\title{
Discrete mathematical modeling and optimal control of the marital status: the monogamous marriage case
}

\author{
Mustapha Lhous ${ }^{1 *}$, Mostafa Rachik ${ }^{2}$, Hassan Laarabi ${ }^{2}$ and Ahmed Abdelhak ${ }^{3}$
}

\author{
"Correspondence: \\ mlhous17@gmail.com \\ 'Laboratory of Modelling, Analysis, \\ Control and Statistics, Department \\ of Mathematics and Computer \\ Science, Faculty of Sciences Ain \\ Chock, Hassan II University of \\ Casablanca, B.P 5366 Maarif, \\ Casablanca, Morocco \\ Full list of author information is \\ available at the end of the article
}

\begin{abstract}
In this paper, we consider a discrete model of the marital status of the family dynamics. It is assumed that individuals in the society can be classed in one of the eight compartments: virgin men, virgin women, married men, married women, divorced men, divorced women, widowed men and widowed women. The objective of this work is to treat the modeling and control the system that describes the case of monogamous marriage. We determine two controls which allow to reduce the number of virgin, divorced individuals and increase the number of married individuals. The first control is the benefits of an awareness campaign to educate virgin men and women about the benefits of marriage for the individual and for the society, the second control characterizes the legal procedures, administrative complications and the heavy financial and social consequences of divorces. The optimal control problems are procured based on a discrete version of Pontryagin's maximum principle and determined numerically using a progressive-regressive discrete schema that converges following a convenient test related to the Forward-Backward Sweep Method (FBSM) on the optimal control.
\end{abstract}

Keywords: discrete marital status model; monogamous marriage; Pontryagin's maximum principle; optimal control

\section{Introduction}

There are different categories of marriage. The most recognized one is the monogamous marriage, when a man marries a single woman. The second type of marriage is polygamy, it is the fact that a man marries several women. Lately another type of marriage has emerged, it is the marriage of homosexuals, it is the union of two people of the same sex. In this work we will treat the modeling and control of a system that describes the case of monogamous marriage.

Mathematical models are tools for understanding the functioning of natural systems and for predicting their evolution. Among these models, there are those that study the dynamics of populations and ecosystems. Many researchers have studied models of population dynamics: prey and predator dynamics [1-4], epidemic dynamics in a population [5-8], molecular systems [9-11], etc.

During longitudinal studies [12-19] and [20], changes in mental, physical, moral and social functioning were assessed of married, unmarried and widowed women. These studies

(c) The Author(s) 2017. This article is distributed under the terms of the Creative Commons Attribution 4.0 International License (http://creativecommons.org/licenses/by/4.0/), which permits unrestricted use, distribution, and reproduction in any medium, provided you give appropriate credit to the original author(s) and the source, provide a link to the Creative Commons license, and indicate if changes were made. 
have determined that health effects differ between never-married women, divorced and separated women. Marriage can be beneficial to health because many spouses monitor and attempt to control their spouses' health behaviors. There is support for social behavior and health behavior among married couples.

Following the modeling of the dynamics of the population and inspired by population census studies in different countries, and in particular by the official results of the 2014 population census in Morocco prepared by the High Commissioner for Planning (HCP, public institution) [21], which provided statistical data on the evolution of the demographic and socio-economic situation of women, we are interested in this work in defining a model that presents the evolution of the civil state of a population.

Civil status is the situation of a person in the family and society. A person's marital status is positioned in one of four categories: virgin, married, divorced or widowed. The model VMDW to be studied classifies the marital status of the family dynamics of a population into eight compartments: virgin men $V^{M}$, virgin women $V^{W}$, married men $M^{M}$, married women $M^{W}$, divorced men $D^{M}$, divorced women $D^{W}$, widowed men $W^{M}$ and widowed women $W^{W}$.

The official results of Morocco's population census for 2014, presented by HCP, show that the so-called final celibacy rate, i.e., those who did not get married even though they reached the age of 55, is $5.9 \%$ of the total number of singles, while in 2004 it was only $3 \%$. The results of 33.8 million Moroccans, including 86,000 foreigners, showed that the percentage of women who never married, despite their age-old proportion, was $6.7 \%$, while men's rate was $5.1 \%$. The phenomenon of celibacy at the age of 55 appears more in urban areas than cities. However, the news of spinsterhood or celibacy did not all rise. The results showed that the proportion of single and married women who reached or exceeded the age of 15 (the official marriage age in Morocco is 18 years old) declined from $39.7 \%$ in 2004 to $34.8 \%$ in 2014 . In contrast, the proportion of married couples rose to $57.5 \%$, and the proportion of divorced persons rose to $2.2 \%$, after not marrying $2 \%$ in 2004. The results indicated the age at the first marriage, which was 25.8 years for women and 31.4 years for men. In the case of persons with special needs, there was a clear rise up to $23.5 \%$ in the number of widows among them, considering that the proportion of married persons among them was $45.6 \%$, and the percentage of divorcees was $2.6 \%$.

According to the official data of the HCP, in Morocco, 6.5 million young people aged 15 to 29 are unmarried, this rate varies according to the age groups, for the 20 to 24 years (76.2\%) and 25 to 29 years (54\%). Regarding the divorce rate, the HCP indicates that it decreased between 30\% and 10\% during 1960 and 2010, and varies according to the duration of marriage. Thus for couples with 20 years of common life, the divorce rate decreases to $3 \%$, and for those with 5 years of marriage, it is more than $30 \%$.

Among the key numbers, $7 \%$ of married women to distant cousins or relatives have at least one divorce. $11.3 \%$ of women who have no family ties with their spouses are divorced. $19.8 \%$ of women without children experienced divorce. Women with at least one boy divorce half as much as the others $(8.8 \%) .11 .5 \%$ is the divorce rate among uneducated women. $5.8 \%$ of educated women know about divorce.

Using these statistical studies and the evolution of marital status, we define a discrete model VMDW which defines the evolution of the marital status of the marriage in a population. In this article we are interested in the control of this system. The controls have been established to reduce the number of unmarried and divorced individuals and to increase 
the number of married individuals. The first control was considered as an accompaniment to the sensitization of the individuals to the benefit of marriage for the psychic and social stability of persons and also for the society. The second control is determined for the persons who have initiated the divorce proceedings. This control is considered as a long and costly legal procedure. As we know, mathematics is an abstract tool that is used to solve real problems. In our work we assume (but we are not obliged to do so) that the control theory is more appropriate to solve our problem. In recent years, many attempts have been made to develop the control strategy for different systems [5, 22-27] and [28]. There are a number of different methods for calculating the optimal control for a specific mathematical model. For example, Pontryagin's maximum principle [29] allows the calculation of the optimal control for an ordinary equation model system with a given constraint.

In this paper, we investigate an approach that determines an optimal control relative to a discrete marital status model which allows to inoculate a control (awareness), which allows to reduce the virgin individuals and to increase the number of married individuals with a minimal cost. The optimal control problem was subject of an optimization criterion represented by the minimization of an objective function. The optimality system is solved based on an iterative discrete schema that converges following an appropriate test similar to the one related to the Forward-Backward Sweep Method (FBSM).

The paper is organized as follows. In Section 2, the model VMDW is described. In Section 3, we give some results concerning the existence of the optimal control, and we use Pontryagin's maximum principle to perform the analysis of control strategies and to determine the necessary condition for the optimal control. Numerical simulations are given in Section 4. Finally, we conclude the paper in Section 5.

\section{Model description}

We consider a discrete-time model VMDW of the marital status of the family dynamic within a domain of interest $\Omega$, which represents a country, a city, a town, or a small domain. The population marital status associated to a domain $\Omega$ is noted by the state $V_{i}^{M}$, $V_{i}^{W}, M_{i}^{M}, M_{i}^{W}, D_{i}^{M}, D_{i}^{W}, W_{i}^{M}$ and $W_{i}^{W}$. The unit of $i$ can correspond to days, months or years, it depends on the frequency of the survey and demographic studies as needed. However, demographic statistics are generally done annually, so the unit of $i$ can be considered as years. The following system describes a discrete model of the marital status of the monogamous marriage case:

$$
\begin{aligned}
V_{i+1}^{M}= & V_{i}^{M}-\frac{\alpha_{1} V_{i}^{W}+\beta_{1} D_{i}^{W}+\alpha_{3} W_{i}^{W}}{N_{i}} V_{i}^{M}, \\
V_{i+1}^{W}= & V_{i}^{W}-\frac{\alpha_{1} V_{i}^{M}+\beta_{2} D_{i}^{M}+\alpha_{2} W_{i}^{M}}{N_{i}} V_{i}^{W}, \\
M_{i+1}^{M}= & M_{i}^{M}+\frac{\alpha_{1} V_{i}^{W}+\beta_{1} D_{i}^{W}+\alpha_{3} W_{i}^{W}}{N_{i}} V_{i}^{M}+\frac{\beta_{2} V_{i}^{W}+\beta_{3} D_{i}^{W}+\beta_{5} W_{i}^{W}}{N_{i}} D_{i}^{M} \\
& +\frac{\alpha_{2} V_{i}^{W}+\beta_{4} D_{i}^{W}+\alpha_{4} W_{i}^{W}}{N_{i}} W_{i}^{M}-\left(\lambda_{1}+\lambda_{2}\right) M_{i}^{M}, \\
M_{i+1}^{W}= & M_{i}^{W}+\frac{\alpha_{1} V_{i}^{M}+\beta_{2} D_{i}^{M}+\alpha_{2} W_{i}^{M}}{N_{i}} V_{i}^{W}+\frac{\beta_{1} V_{i}^{M}+\beta_{3} D_{i}^{M}+\beta_{4} W_{i}^{M}}{N_{i}} D_{i}^{W} \\
& +\frac{\alpha_{2} V_{i}^{W}+\beta_{4} D_{i}^{W}+\alpha_{4} W_{i}^{W}}{N_{i}} W_{i}^{M}-\left(\lambda_{1}+\lambda_{3}\right) M_{i}^{W},
\end{aligned}
$$




$$
\begin{aligned}
& D_{i+1}^{M}=D_{i}^{M}-\frac{\beta_{2} V_{i}^{W}+\beta_{3} D_{i}^{W}+\beta_{5} W_{i}^{W}}{N_{i}} D_{i}^{M}+\lambda_{1} M_{i}^{M}, \\
& D_{i+1}^{W}=D_{i}^{W}-\frac{\beta_{1} V_{i}^{M}+\beta_{3} D_{i}^{M}+\beta_{4} W_{i}^{M}}{N_{i}} D_{i}^{W}+\lambda_{1} M_{i}^{W}, \\
& W_{i+1}^{M}=W_{i}^{M}-\frac{\alpha_{2} V_{i}^{W}+\beta_{4} D_{i}^{W}+\alpha_{4} W_{i}^{W}}{N_{i}} W_{i}^{M}+\lambda_{3} M_{i}^{W}, \\
& W_{i+1}^{W}=W_{i}^{W}-\frac{\alpha_{3} V_{i}^{M}+\beta_{5} D_{i}^{M}+\alpha_{4} W_{i}^{M}}{N_{i}} W_{i}^{W}+\lambda_{2} M_{i}^{M},
\end{aligned}
$$

where $V_{0}^{M}, V_{0}^{W}, M_{0}^{M}, M_{0}^{W}, D_{0}^{M}, D_{0}^{W}, W_{0}^{M}$ and $W_{0}^{W}$ are the given initial states. In equations (1)-(8), all parameters are nonnegative and defined in Table 1. Schematically, the flow between compartments VMDW is represented in Figure 1.

For equation (1) of the model, virgin men can contact a virgin, divorced or widowed woman with $\alpha_{1}, \beta_{1}$ and $\alpha_{3}$ rates respectively, and this contact can result in a marriage. Thus the number of virgin men decreases and the number of virgins at the instant $i$ is

Table 1 The description of parameters used for the definition of discrete time systems (1)-(8)

\begin{tabular}{ll}
\hline Parameter & Description \\
\hline$\alpha_{1}$ & Marriage rate of a virgin man to a virgin woman \\
$\alpha_{2}$ & Marriage rate of a widower to a virgin woman \\
$\alpha_{3}$ & Marriage rate of a virgin man to a widow \\
$\alpha_{4}$ & Marriage rate of a widower to a widow \\
$\beta_{1}$ & Marriage rate of a virgin man to a divorced woman \\
$\beta_{2}$ & Marriage rate of a divorced man to a virgin woman \\
$\beta_{3}$ & Marriage rate of a divorced man to a divorced woman \\
$\beta_{4}$ & Marriage rate of a widower to a divorced woman \\
$\beta_{5}$ & Marriage rate of a divorced man to a widow \\
$\lambda_{1}$ & Divorce rate of married men \\
$\lambda_{2}$ & Widow rate of married women \\
$\lambda_{3}$ & Widow rate of married men \\
\hline
\end{tabular}

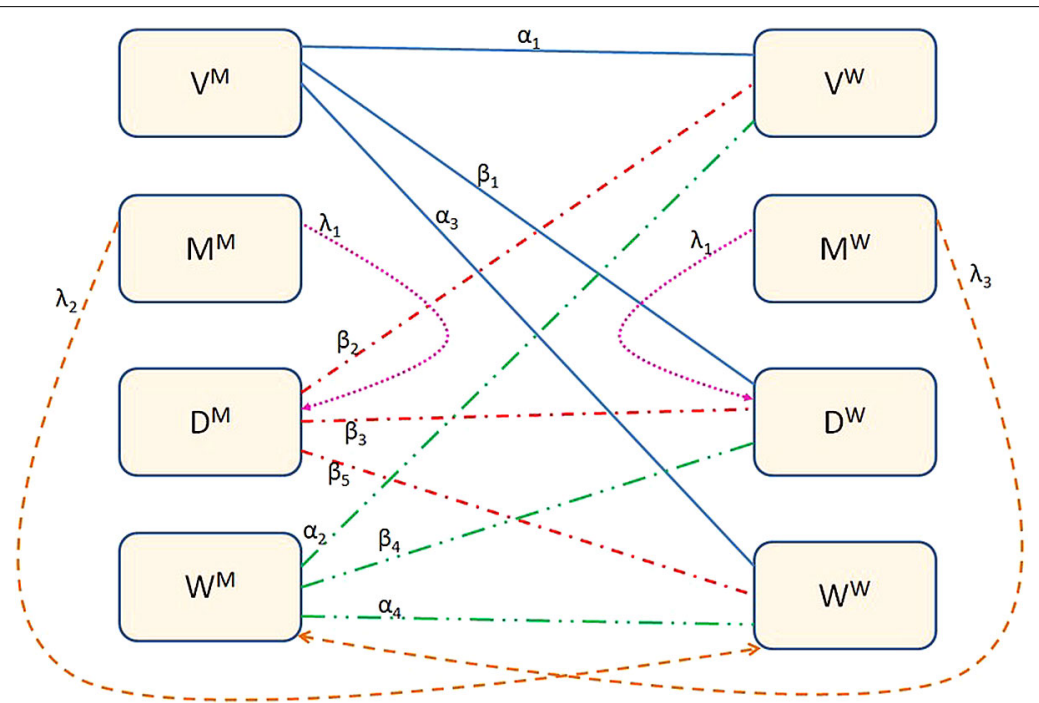

Figure 1 The flow between the eight compartments VMDW. 
substituted for the number $\alpha_{1} \frac{V_{i}^{M} V_{i}^{W}}{N_{i}}+\beta_{1} \frac{V_{i}^{M} D_{i}^{W}}{N_{i}}+\alpha_{3} \frac{V_{i}^{M} W_{i}^{W}}{N_{i}}$ and this number is added to the number of men marrying at the time $i+1$.

Similarly, in equation (2) the number of virgin women decreases at the instant $i+1$ by substituting the number of virgin women at the instant $i$ with the number $\alpha_{1} \frac{V_{i}^{M} V_{i}^{W}}{N_{i}}+$ $\beta_{2} \frac{D_{i}^{M} V_{i}^{W}}{N_{i}}+\alpha_{2} \frac{W_{i}^{M} V_{i}^{W}}{N_{i}}$ which represents the number of married women after the contact with a virgin, divorced or widowed man with the $\alpha_{1}, \beta_{2}$ and $\alpha_{2}$ levels respectively.

In equation (5), a divorced man can contact a virgin, divorced or widowed woman with $\beta_{2}, \beta_{3}$ and $\beta_{5}$ rates respectively, so this contact can result in marriage. Then the number $\beta_{2} \frac{D_{i}^{M} V_{i}^{W}}{N_{i}}+\beta_{3} \frac{D_{i}^{M} D_{i}^{W}}{N_{i}}+\beta_{5} \frac{D_{i}^{M} W_{i}^{W}}{N_{i}}$ is decreased by the number of divorced men the time $i+1$ and added to the number of married men. In addition a married man can divorce with a rate of $\lambda_{1}$, and as a result, the number of divorced men increases from $\lambda_{1} M_{i}^{M}$. The same principle applies to equations (6), (7) and (8).

For equation (3) the number of married men increases at the instant $i+1$ by the number of virgin, divorced and widowed men who are married by contacting virgin, divorced or widowed women and decreases the natural mortality with $\lambda_{2}$ and the divorce rate with $\lambda_{1}$ rate. The same principle can be applied to equations (4).

In equations (3) and (4) from the model, the natural mortality of married men and women was considered. The mortality rate for married men is $\lambda_{2}$ and the mortality rate for married women is $\lambda_{3}$. These rates appear in equations (7) and (8) which correspond to widowed men and women, respectively, so the number of mortalities, which is $\lambda_{2} M_{i}^{M}$ for married men, is added to the number of widowed women, and similarly the number $\lambda_{3} M_{i}^{W}$ is added to the number of widowed men.

\section{An optimal control problem}

Optimal control approach has been applied to models (1)-(8) to reduce the number of virgin, divorced individuals and increase the number of married individuals along the control strategy period. For this, we introduce a control variable $\left(u_{i}, v_{i}\right)$ that characterizes the benefits of an awareness campaign to educate virgin men and women about the benefits of marriage for the individual and for the society, especially the legal procedures, administrative complications, the heavy financial and social consequences of divorces respectively in the above mentioned model (1)-(8). Then the model is given by the following equations:

$$
\begin{aligned}
V_{i+1}^{M}= & V_{i}^{M}-\frac{\alpha_{1} V_{i}^{W}+\beta_{1} D_{i}^{W}+\alpha_{3} W_{i}^{W}}{N_{i}} V_{i}^{M}-u_{i} V_{i}^{M}, \\
V_{i+1}^{W}= & V_{i}^{W}-\frac{\alpha_{1} V_{i}^{M}+\beta_{2} D_{i}^{M}+\alpha_{2} W_{i}^{M}}{N_{i}} V_{i}^{W}-u_{i} V_{i}^{W}, \\
M_{i+1}^{M}= & M_{i}^{M}+\frac{\alpha_{1} V_{i}^{W}+\beta_{1} D_{i}^{W}+\alpha_{3} W_{i}^{W}}{N_{i}} V_{i}^{M}+\frac{\beta_{2} V_{i}^{W}+\beta_{3} D_{i}^{W}+\beta_{5} W_{i}^{W}}{N_{i}} D_{i}^{M} \\
& +\frac{\alpha_{2} V_{i}^{W}+\beta_{4} D_{i}^{W}+\alpha_{4} W_{i}^{W}}{N_{i}} W_{i}^{M}-\left(\lambda_{1}+\lambda_{2}\right) M_{i}^{M}+u_{i} V_{i}^{M}+v_{i} D_{i}^{M}, \\
M_{i+1}^{W}= & M_{i}^{W}+\frac{\alpha_{1} V_{i}^{M}+\beta_{2} D_{i}^{M}+\alpha_{2} W_{i}^{M}}{N_{i}} V_{i}^{W}+\frac{\beta_{1} V_{i}^{M}+\beta_{3} D_{i}^{M}+\beta_{4} W_{i}^{M}}{N_{i}} D_{i}^{W} \\
& +\frac{\alpha_{2} V_{i}^{W}+\beta_{4} D_{i}^{W}+\alpha_{4} W_{i}^{W}}{N_{i}} W_{i}^{M}-\left(\lambda_{1}+\lambda_{3}\right) M_{i}^{W}+u_{i} V_{i}^{W}+v_{i} D_{i}^{W}, \\
D_{i+1}^{M}= & D_{i}^{M}-\frac{\beta_{2} V_{i}^{W}+\beta_{3} D_{i}^{W}+\beta_{5} W_{i}^{W}}{N_{i}} D_{i}^{M}+\lambda_{1} M_{i}^{M}-v_{i} D_{i}^{M},
\end{aligned}
$$




$$
\begin{aligned}
& D_{i+1}^{W}=D_{i}^{W}-\frac{\beta_{1} V_{i}^{M}+\beta_{3} D_{i}^{M}+\beta_{4} W_{i}^{M}}{N_{i}} D_{i}^{W}+\lambda_{1} M_{i}^{W}-v_{i} D_{i}^{W}, \\
& W_{i+1}^{M}=W_{i}^{M}-\frac{\alpha_{2} V_{i}^{W}+\beta_{4} D_{i}^{W}+\alpha_{4} W_{i}^{W}}{N_{i}} W_{i}^{M}+\lambda_{3} M_{i}^{W}, \\
& W_{i+1}^{W}=W_{i}^{W}-\frac{\alpha_{3} V_{i}^{M}+\beta_{5} D_{i}^{M}+\alpha_{4} W_{i}^{M}}{N_{i}} W_{i}^{W}+\lambda_{2} M_{i}^{M} .
\end{aligned}
$$

The model (9)-(16) fulfils the constant population through time constraint, i.e.,

$$
N_{i}=V_{i}^{M}+V_{i}^{W}+M_{i}^{M}+M_{i}^{W}+D_{i}^{M}+D_{i}^{W}+W_{i}^{M}+W_{i}^{W}=N>0
$$

Note that this assertion proves that the constant population through time is independent of the control strategy.

\subsection{Characterization of the optimal control}

For an initial state $\left(V_{0}^{M}, V_{0}^{W}, M_{0}^{M}, M_{0}^{W}, D_{0}^{M}, D_{0}^{W}, W_{0}^{M}, W_{0}^{W}\right)$, we consider an optimization criterion defined by the following objective function:

$$
\begin{aligned}
J(u, v)= & \sum_{i=0}^{N}\left(A_{1} V_{i}^{W}+A_{2} D_{i}^{W}-A_{3} M_{i}^{W}\right) \\
& +\sum_{i=0}^{N-1}\left(\frac{\tau_{1}}{2}\left(u_{i}\right)^{2}+\frac{\tau_{2}}{2}\left(v_{i}\right)^{2}\right)
\end{aligned}
$$

subject to system (9)-(16). Here $A_{1}, A_{2}$ and $A_{3}$ are positive constants to keep a balance in the size of $V_{i}^{W}, D_{i}^{W}$ and $M_{i}^{W}$, respectively. In the objective functional, $\tau_{1}$ and $\tau_{2}$ are the positive weight parameters which are associated with the controls $u_{i}$ and $v_{i}$.

In other words, we seek the optimal controls $\left(u^{*}, v^{*}\right)$ such that

$$
J\left(u^{*}, v^{*}\right)=\min \left\{J(u, v) \mid(u, v) \in \mathcal{U}_{\mathrm{ad}}\right\}
$$

where $\mathcal{U}_{\text {ad }}$ is the set of admissible controls defined by

$$
\mathcal{U}_{\mathrm{ad}}=\left\{(u, v) \mid u^{\min } \leq u_{i} \leq u^{\max }, v^{\min } \leq v_{i} \leq v^{\max }, i \in\{0 \ldots, N-1\}\right\}
$$

where $\left.\left(u^{\min }, u^{\max }, v^{\min }, v^{\max }\right) \in\right] 0,1\left[^{4}\right.$.

The sufficient condition for the existence of an optimal control $\left(u^{*}, v^{*}\right)$ for problem (17) comes from the following theorem.

Theorem 1 There exists an optimal control $\left(u^{*}, v^{*}\right)$ such that

$$
J\left(u^{*}, v^{*}\right)=\min _{(u, v) \in \mathcal{U}_{\mathrm{ad}}} J(u, v)
$$

subject to the control system (9)-(16) with initial conditions.

Proof There are a finite number of time steps, $V^{M}=\left(V_{0}^{M}, V_{1}^{M}, \ldots, V_{N}^{M}\right), V^{W}=\left(V_{0}^{W}, V_{1}^{W}\right.$, $\left.\ldots, V_{N}^{W}\right), \ldots, W^{W}=\left(W_{0}^{W}, W_{1}^{W}, \ldots, W_{N}^{W}\right)$ are uniformly bounded for all $(u, v) \in \mathcal{U}_{\mathrm{ad}}$. Thus 
$J(u, v)$ is uniformly bounded for all $(u, v)$ in the control set $\mathcal{U}_{\text {ad }}$. Since $J(u, v)$ is bounded, $\inf _{(u, v) \in \mathcal{U}_{\mathrm{ad}}} J(u, v)$ is finite, and there exists a sequence $\left(u^{j}, v^{j}\right) \in \mathcal{U}_{\mathrm{ad}}$ such that $\lim _{j \rightarrow \infty} J\left(u^{j}\right.$, $\left.v^{j}\right)=\inf _{(u, v) \in \mathcal{U}_{\mathrm{ad}}} J(u, v)$ and corresponding sequences of states $V^{M^{j}}, V^{W^{j}}, \ldots, W^{W^{j}}$. Since there is a finite number of uniformly bounded sequences, there exist $\left(u^{*}, v^{*}\right) \in \mathcal{U}_{\mathrm{ad}}$ and $V^{M^{j}}, \ldots, W^{W^{j}} \in \mathbb{R}^{N+1}$ such that on a subsequence, $u^{j} \longrightarrow u^{*}, v^{j} \longrightarrow v^{*}, V^{M^{j}} \longrightarrow$ $V^{M^{*}}, \ldots, W^{W^{j}} \rightarrow W^{W^{*}}$. Finally, due to the finite dimensional structure of system (9)(16) and the objective function $J(u, v),\left(u^{*}, v^{*}\right)$ is an optimal control with corresponding states $V^{M^{*}}, V^{W^{*}}, \ldots, W^{W^{*}}$. Therefore $\inf _{(u, v) \in \mathcal{U}_{\mathrm{ad}}} J(u, v)$ is achieved.

In order to find an optimal solution, first we find the Hamiltonian for the optimal control problem (17). In fact, the Hamiltonian $H$ of the optimal problem is given by

$$
\begin{aligned}
H= & A_{1} V_{i}^{W}+A_{2} D_{i}^{W}-A_{3} M_{i}^{W}+\frac{\tau_{1}}{2} u_{i}^{2}+\frac{\tau_{2}}{2} v_{i}^{2} \\
& +\zeta_{1, i+1}\left[V_{i}^{M}-\frac{\alpha_{1} V_{i}^{W}+\beta_{1} D_{i}^{W}+\alpha_{3} W_{i}^{W}}{N_{i}} V_{i}^{M}-u_{i} V_{i}^{M}\right] \\
& +\zeta_{2, i+1}\left[V_{i}^{W}-\frac{\alpha_{1} V_{i}^{M}+\beta_{2} D_{i}^{M}+\alpha_{2} W_{i}^{M}}{N_{i}} V_{i}^{W}-u_{i} V_{i}^{W}\right] \\
& +\zeta_{3, i+1}\left[M_{i}^{M}+\frac{\alpha_{1} V_{i}^{W}+\beta_{1} D_{i}^{W}+\alpha_{3} W_{i}^{W}}{N_{i}} V_{i}^{M}+\frac{\beta_{2} V_{i}^{W}+\beta_{3} D_{i}^{W}+\beta_{5} W_{i}^{W}}{N_{i}} D_{i}^{M}\right. \\
& \left.+\frac{\alpha_{2} V_{i}^{W}+\beta_{4} D_{i}^{W}+\alpha_{4} W_{i}^{W}}{N_{i}} W_{i}^{M}-\left(\lambda_{1}+\lambda_{2}\right) M_{i}^{M}+u_{i} V_{i}^{M}+v_{i} D_{i}^{M}\right] \\
& +\zeta_{4, i+1}\left[M_{i}^{W}+\frac{\alpha_{1} V_{i}^{M}+\beta_{2} D_{i}^{M}+\alpha_{2} W_{i}^{M}}{N_{i}} V_{i}^{W}+\frac{\beta_{1} V_{i}^{M}+\beta_{3} D_{i}^{M}+\beta_{4} W_{i}^{M}}{N_{i}} D_{i}^{W}\right. \\
& \left.+\frac{\alpha_{2} V_{i}^{W}+\beta_{4} D_{i}^{W}+\alpha_{4} W_{i}^{W}}{N_{i}} W_{i}^{M}-\left(\lambda_{1}+\lambda_{3}\right) W_{i}^{M}+u_{i} V_{i}^{W}+v_{i} D_{i}^{W}\right] \\
& +\zeta_{5, i+1}\left[D_{i}^{M}-\frac{\beta_{2} V_{i}^{W}+\beta_{3} D_{i}^{W}+\beta_{5} W_{i}^{W}}{N_{i}} D_{i}^{M}+\lambda_{1} M_{i}^{M}-v_{i} D_{i}^{M}\right] \\
& +\zeta_{6, i+1}\left[D_{i}^{W}-\frac{\beta_{1} V_{i}^{M}+\beta_{3} D_{i}^{M}+\beta_{4} W_{i}^{M}}{N_{i}} D_{i}^{W}+\lambda_{1} M_{i}^{W}-v_{i} D_{i}^{W}\right] \\
& +\zeta_{7, i+1}\left[W_{i}^{M}-\frac{\alpha_{2} V_{i}^{W}+\beta_{4} D_{i}^{W}+\alpha_{4} W_{i}^{W}}{N_{i}} W_{i}^{M}+\lambda_{3} M_{i}^{W}\right] \\
& +\zeta_{8, i+1}\left[W_{i}^{W}-\frac{\alpha_{3} V_{i}^{M}+\beta_{5} D_{i}^{M}+\alpha_{4} W_{i}^{M}}{N_{i}} W_{i}^{W}+\lambda_{2} M_{i}^{M}\right] \\
&
\end{aligned}
$$

where $\zeta_{1}, \zeta_{2}, \ldots, \zeta_{8}$ are the adjoint functions to be determined suitably.

At the same time, by using Pontryagin's maximum principle [29], we derive necessary conditions for our optimal control. We obtain the following theorem.

Theorem 2 (Necessary conditions) Let $V^{M^{*}}, V^{W^{*}}, M^{M^{*}}, M^{W^{*}}, D^{M^{*}}, D^{W^{*}}, W^{M^{*}}$ and $W^{W^{*}}$ be optimal state solutions with an associated optimal control $\left(u^{*}, v^{*}\right)$ for the optimal control problem (17). Then there exist adjoint variables $\zeta_{1}, \zeta_{2}, \ldots, \zeta_{8}$, that satisfy

$$
\begin{aligned}
\Delta \zeta_{1, i}= & -\zeta_{1, i+1}+\alpha_{1} V_{i}^{W}\left(\zeta_{1, i+1}+\zeta_{2, i+1}-\zeta_{3, i+1}-\zeta_{4, i+1}\right) \\
& +\alpha_{3} W_{i}^{W}\left(\zeta_{1, i+1}-\zeta_{3, i+1}-\zeta_{4, i+1}+\zeta_{8, i+1}\right) \\
& +\beta_{1} D_{i}^{W}\left(\zeta_{1, i+1}-\zeta_{3, i+1}-\zeta_{4, i+1}+\zeta_{6, i+1}\right)+u_{i}\left(\zeta_{1, i+1}-\zeta_{3, i+1}\right)
\end{aligned}
$$




$$
\begin{aligned}
\Delta \zeta_{2, i}= & -A_{1}-\zeta_{2, i+1}+\alpha_{1} V_{i}^{M}\left(\zeta_{1, i+1}+\zeta_{2, i+1}-\zeta_{3, i+1}-\zeta_{4, i+1}\right) \\
& +\alpha_{2} W_{i}^{M}\left(\zeta_{2, i+1}-\zeta_{3, i+1}-\zeta_{4, i+1}+\zeta_{7, i+1}\right) \\
& +\beta_{2} D_{i}^{M}\left(\zeta_{2, i+1}-\zeta_{3, i+1}-\zeta_{4, i+1}+\zeta_{5, i+1}\right)+u_{i}\left(\zeta_{2, i+1}-\zeta_{4, i+1}\right) \\
\Delta \zeta_{3, i}= & -\zeta_{3, i+1}+\lambda_{1}\left(\zeta_{3, i+1}-\zeta_{5, i+1}\right)+\lambda_{2}\left(\zeta_{3, i+1}-\zeta_{8, i+1}\right) \\
\Delta \zeta_{4, i}= & A_{3}-\zeta_{4, i+1}+\lambda_{1}\left(\zeta_{4, i+1}-\zeta_{6, i+1}\right)+\lambda_{3}\left(\zeta_{4, i+1}-\zeta_{7, i+1}\right) \\
\Delta \zeta_{5, i}= & -\zeta_{5, i+1}+\beta_{2} V_{i}^{W}\left(\zeta_{2, i+1}-\zeta_{3, i+1}-\zeta_{4, i+1}+\zeta_{5, i+1}\right) \\
& +\alpha_{3} D_{i}^{W}\left(-\zeta_{3, i+1}-\zeta_{4, i+1}+\zeta_{5, i+1}+\zeta_{6, i+1}\right) \\
& +\beta_{5} W_{i}^{W}\left(-\zeta_{3, i+1}-\zeta_{4, i+1}+\zeta_{5, i+1}+\zeta_{8, i+1}\right)+v_{i}\left(-\zeta_{3, i+1}+\zeta_{5, i+1}\right) \\
\Delta \zeta_{6, i}= & -A_{2}-\zeta_{6, i+1}+\beta_{1} V_{i}^{M}\left(\zeta_{1, i+1}-\zeta_{3, i+1}-\zeta_{4, i+1}+\zeta_{6, i+1}\right) \\
& +\alpha_{3} D_{i}^{M}\left(-\zeta_{3, i+1}-\zeta_{4, i+1}+\zeta_{5, i+1}+\zeta_{6, i+1}\right) \\
& +\beta_{4} W_{i}^{M}\left(-\zeta_{3, i+1}-\zeta_{4, i+1}+\zeta_{6, i+1}+\zeta_{7, i+1}\right)+v_{i}\left(-\zeta_{4, i+1}+\zeta_{6, i+1}\right) \\
\Delta \zeta_{8, i}= & -\zeta_{8, i+1}+\alpha_{3} V_{i}^{M}\left(\zeta_{1, i+1}-\zeta_{3, i+1}-\zeta_{4, i+1}+\zeta_{8, i+1}\right) \\
& +\alpha_{4} W_{i}^{M}\left(-\zeta_{3, i+1}-\zeta_{4, i+1}+\zeta_{7, i+1}+\zeta_{8, i+1}\right) \\
& +\beta_{5} D_{i}^{M}\left(-\zeta_{3, i+1}-\zeta_{4, i+1}+\zeta_{5, i+1}+\zeta_{8, i+1}\right) \\
\Delta \zeta_{7, i}= & -\zeta_{7, i+1}+\alpha_{2} V_{i}^{W}\left(\zeta_{2, i+1}-\zeta_{3, i+1}-\zeta_{4, i+1}+\zeta_{7, i+1}\right) \\
& +\alpha_{4} W_{i}^{W}\left(-\zeta_{3, i+1}-\zeta_{4, i+1}+\zeta_{7, i+1}+\zeta_{8, i+1}\right) \\
& \beta_{4} D_{i}^{W}\left(-\zeta_{3, i+1}-\zeta_{4, i+1}+\zeta_{6, i+1}+\zeta_{7, i+1}\right) \\
& \\
& \\
&
\end{aligned}
$$

with transversality conditions

$$
\begin{aligned}
& \zeta_{1, N}=0, \quad \zeta_{2, N}=A_{1}, \quad \zeta_{3, N}=0, \quad \zeta_{4, N}=-A_{3}, \\
& \zeta_{5, N}=0, \quad \zeta_{6, N}=A_{2}, \quad \zeta_{7, N}=0 \quad \text { and } \quad \zeta_{8, N}=0 .
\end{aligned}
$$

Furthermore, the optimal control $\left(u_{i}{ }^{*}, v_{i}{ }^{*}\right)$ is given by

$$
\begin{aligned}
& u_{i}^{*}=\min \left\{\max \left\{\frac{V_{i}^{M}\left(\zeta_{1, i+1}-\zeta_{3, i+1}\right)+D_{i}^{W}\left(\zeta_{2, i+1}-\zeta_{4, i+1}\right)}{\tau_{1}}, u_{\min }\right\}, u_{\max }\right\}, \\
& v_{i}^{*}=\min \left\{\max \left\{\frac{D_{i}^{M}\left(\zeta_{5, i+1}-\zeta_{3, i+1}\right)+D_{i}^{W}\left(\zeta_{6, i+1}-\zeta_{4, i+1}\right)}{\tau_{2}}, u_{\min }\right\}, u_{\max }\right\}
\end{aligned}
$$

for $i=0, \ldots, N-1$.

Proof Using Pontryagin's maximum principle [29] and setting $V^{M^{*}}, V^{W^{*}}, M^{M^{*}}, M^{W^{*}}$, $D^{M^{*}}, D^{W^{*}}, W^{M^{*}}, W^{W^{*}}$ and $\left(u^{*}, v^{*}\right)$, we obtain the following adjoint equations:

$$
\begin{aligned}
\Delta \zeta_{1, i}= & -\frac{\partial H}{\partial V_{i}^{M}} \\
= & -\left[\zeta_{1, i+1}\left(1-\alpha_{1} V_{i}^{W}-\alpha_{3} W_{i}^{W}-\beta_{1} D_{i}^{W}-u_{i}\right)-\zeta_{2, i+1} \alpha_{1} V_{i}^{W}\right. \\
& +\zeta_{3, i+1}\left(\alpha_{1} V_{i}^{W}+\beta_{1} D_{i}^{W}+\alpha_{3} W_{i}^{W}+u_{i}\right)+\zeta_{4, i+1}\left(\alpha_{1} V_{i}^{W}+\beta_{1} D_{i}^{W}\right.
\end{aligned}
$$




$$
\begin{aligned}
& \left.\left.+\alpha_{3} W_{i}^{W}+u_{i}\right)-\zeta_{6, i+1} \beta_{1} D_{i}^{W}-\zeta_{8, i+1} \alpha_{3} W_{i}^{W}\right] \\
& =-\zeta_{1, i+1}+\alpha_{1} V_{i}^{W}\left(\zeta_{1, i+1}+\zeta_{2, i+1}-\zeta_{3, i+1}-\zeta_{4, i+1}\right) \\
& +\alpha_{3} W_{i}^{W}\left(\zeta_{1, i+1}-\zeta_{3, i+1}-\zeta_{4, i+1}+\zeta_{8, i+1}\right) \\
& +\beta_{1} D_{i}^{W}\left(\zeta_{1, i+1}-\zeta_{3, i+1}-\zeta_{4, i+1}+\zeta_{6, i+1}\right)+u_{i}\left(\zeta_{1, i+1}-\zeta_{3, i+1}\right) \\
& \Delta \zeta_{2, i}=-\frac{\partial H}{\partial V_{i}^{W}} \\
& =-\left[A_{1}-\zeta_{1, i+1} \alpha_{1} V_{i}^{M}+\zeta_{2, i+1}\left(1-\alpha_{1} V_{i}^{M}-\beta_{2} D_{i}^{M}-\alpha_{2} W_{i}^{M}-u_{i}\right)\right. \\
& +\zeta_{3, i+1}\left(\alpha_{1} V_{i}^{M}+\beta_{2} D_{i}^{M}+\alpha_{2} W_{i}^{M}\right)+\zeta_{4, i+1}\left(\alpha_{1} V_{i}^{M}+\beta_{2} D_{i}^{M}\right. \\
& \left.\left.+\alpha_{2} W_{i}^{M}+u_{i}\right)-\zeta_{5, i+1} \beta_{2} D_{i}^{M}-\zeta_{7, i+1} \alpha_{2} W_{i}^{M}\right] \\
& =-A_{1}-\zeta_{2, i+1}+\alpha_{1} V_{i}^{M}\left(\zeta_{1, i+1}+\zeta_{2, i+1}-\zeta_{3, i+1}-\zeta_{4, i+1}\right) \\
& +\alpha_{2} W_{i}^{M}\left(\zeta_{2, i+1}-\zeta_{3, i+1}-\zeta_{4, i+1}+\zeta_{7, i+1}\right) \\
& +\beta_{2} D_{i}^{M}\left(\zeta_{2, i+1}-\zeta_{3, i+1}-\zeta_{4, i+1}+\zeta_{5, i+1}\right)+u_{i}\left(\zeta_{2, i+1}-\zeta_{4, i+1}\right), \\
& \Delta \zeta_{3, i}=-\frac{\partial H}{\partial M_{i}^{M}} \\
& =-\left[\zeta_{2, i+1}\left(1-\lambda_{1}-\lambda_{2}\right)+\zeta_{5, i+1} \lambda_{1}+\zeta_{8, i+1} \lambda_{2}\right] \\
& =-\zeta_{3, i+1}+\lambda_{1}\left(\zeta_{3, i+1}-\zeta_{5, i+1}\right)+\lambda_{2}\left(\zeta_{3, i+1}-\zeta_{8, i+1}\right), \\
& \Delta \zeta_{4, i}=-\frac{\partial H}{\partial M_{i}^{W}} \\
& =-\left[-A_{3}+\zeta_{4, i+1}\left(1-\lambda_{1}-\lambda_{3}\right)+\zeta_{6, i+1} \lambda_{1}+\zeta_{7, i+1} \lambda_{3}\right] \\
& =A_{3}-\zeta_{4, i+1}+\lambda_{1}\left(\zeta_{4, i+1}-\zeta_{6, i+1}\right)+\lambda_{3}\left(\zeta_{4, i+1}-\zeta_{7, i+1}\right) \\
& \Delta \zeta_{5, i}=-\frac{\partial H}{\partial D_{i}^{M}} \\
& =-\left[-\zeta_{2, i+1} \beta_{2} V_{i}^{W}+\zeta_{3, i+1}\left(\beta_{2} V_{i}^{W}+\beta_{3} D_{i}^{W}+\beta_{5} W_{i}^{W}+v_{i}\right)\right. \\
& +\zeta_{4, i+1}\left(\beta_{2} V_{i}^{W}+\beta_{3} D_{i}^{W}+\beta_{5} W_{i}^{W}\right)+\zeta_{5, i+1}\left(1-\beta_{2} V_{i}^{W}-\beta_{3} D_{i}^{W}\right. \\
& \left.\left.-\beta_{5} W_{i}^{W}-v_{i}\right)-\zeta_{6, i+1} \beta_{3} D_{i}^{W}-\zeta_{8, i+1} \alpha_{5} W_{i}^{W}\right] \\
& =-\zeta_{5, i+1}+\beta_{2} V_{i}^{W}\left(\zeta_{2, i+1}-\zeta_{3, i+1}-\zeta_{4, i+1}+\zeta_{5, i+1}\right) \\
& +\alpha_{3} D_{i}^{W}\left(-\zeta_{3, i+1}-\zeta_{4, i+1}+\zeta_{5, i+1}+\zeta_{6, i+1}\right) \\
& +\beta_{5} W_{i}^{W}\left(-\zeta_{3, i+1}-\zeta_{4, i+1}+\zeta_{5, i+1}+\zeta_{8, i+1}\right)+v_{i}\left(-\zeta_{3, i+1}+\zeta_{5, i+1}\right) \\
& \Delta \zeta_{6, i}=-\frac{\partial H}{\partial D_{i}^{W}} \\
& =-\left[A_{2}-\zeta_{1, i+1} \beta_{1} D_{i}^{M}+\zeta_{3, i+1}\left(\alpha_{1} V_{i}^{M}+\alpha_{3} D_{i}^{M}+\beta_{4} W_{i}^{M}\right)\right. \\
& +\zeta_{4, i+1}\left(\alpha_{1} V_{i}^{M}+\beta_{3} D_{i}^{M}+\beta_{4} W_{i}^{M}+v_{i}\right)-\zeta_{5, i+1} \beta_{3} D_{i}^{M} \\
& \left.+\zeta_{6, i+1}\left(1-\beta_{1} V_{i}^{M}-\beta_{3} D_{i}^{M}-\beta_{4} W_{i}^{M}-v_{i}\right)-\zeta_{7, i+1} \beta_{4} W_{i}^{M}\right] \\
& =-A_{2}-\zeta_{6, i+1}+\beta_{1} V_{i}^{M}\left(\zeta_{1, i+1}-\zeta_{3, i+1}-\zeta_{4, i+1}+\zeta_{6, i+1}\right)
\end{aligned}
$$




$$
\begin{aligned}
& +\alpha_{3} D_{i}^{M}\left(-\zeta_{3, i+1}-\zeta_{4, i+1}+\zeta_{5, i+1}+\zeta_{6, i+1}\right) \\
& +\beta_{4} W_{i}^{M}\left(-\zeta_{3, i+1}-\zeta_{4, i+1}+\zeta_{6, i+1}+\zeta_{7, i+1}\right)+v_{i}\left(-\zeta_{4, i+1}+\zeta_{6, i+1}\right) \\
\Delta \zeta_{7, i}= & -\frac{\partial H}{\partial W_{i}^{M}} \\
= & -\left[-\zeta_{2, i+1} \alpha_{2} V_{i}^{W}+\zeta_{3, i+1}\left(\alpha_{2} V_{i}^{W}+\alpha_{4} D_{i}^{W}+\alpha_{4} W_{i}^{W}\right)\right. \\
& +\zeta_{4, i+1}\left(\alpha_{2} V_{i}^{W}+\beta_{4} D_{i}^{W}+\alpha_{4} W_{i}^{W}\right)-\zeta_{6, i+1} \beta_{4} D_{i}^{W} \\
& \left.+\zeta_{7, i+1}\left(1-\alpha_{2} V_{i}^{W}-\beta_{4} D_{i}^{W}-\alpha_{4} W_{i}^{W}\right)-\zeta_{8, i+1} \alpha_{4} W_{i}^{W}\right] \\
= & -\zeta_{7, i+1}+\alpha_{2} V_{i}^{W}\left(\zeta_{2, i+1}-\zeta_{3, i+1}-\zeta_{4, i+1}+\zeta_{7, i+1}\right) \\
& +\alpha_{4} W_{i}^{W}\left(-\zeta_{3, i+1}-\zeta_{4, i+1}+\zeta_{7, i+1}+\zeta_{8, i+1}\right) \\
& +\beta_{4} D_{i}^{W}\left(-\zeta_{3, i+1}-\zeta_{4, i+1}+\zeta_{6, i+1}+\zeta_{7, i+1}\right) \\
\Delta \zeta_{8, i}= & -\frac{\partial H}{\partial W_{i}^{W}} \\
= & -\left[-\zeta_{1, i+1} \alpha_{3} V_{i}^{M}+\zeta_{3, i+1}\left(\alpha_{3} V_{i}^{M}+\beta_{5} D_{i}^{M}+\alpha_{4} W_{i}^{M}\right)\right. \\
& +\zeta_{4, i+1}\left(\alpha_{3} V_{i}^{M}+\beta_{5} D_{i}^{M}+\alpha_{4} W_{i}^{M}\right)-\zeta_{5, i+1} \beta_{5} D_{i}^{M} \\
& \left.+\zeta_{8, i+1}\left(1-\alpha_{3} V_{i}^{M}-\beta_{5} D_{i}^{M}-\alpha_{4} W_{i}^{M}\right)-\zeta_{7, i+1} \alpha_{4} W_{i}^{M}\right] \\
= & -\zeta_{8, i+1}+\alpha_{3} V_{i}^{M}\left(\zeta_{1, i+1}-\zeta_{3, i+1}-\zeta_{4, i+1}+\zeta_{8, i+1}\right) \\
& +\alpha_{4} W_{i}^{M}\left(-\zeta_{3, i+1}-\zeta_{4, i+1}+\zeta_{7, i+1}+\zeta_{8, i+1}\right) \\
& +\beta_{5} D_{i}^{M}\left(-\zeta_{3, i+1}-\zeta_{4, i+1}+\zeta_{5, i+1}+\zeta_{8, i+1}\right) \\
&
\end{aligned}
$$

with transversality conditions

$$
\begin{aligned}
& \zeta_{1, N}=0, \quad \zeta_{2, N}=A_{1}, \quad \zeta_{3, N}=0, \quad \zeta_{4, N}=-A_{3}, \\
& \zeta_{5, N}=0, \quad \zeta_{6, N}=A_{2}, \quad \zeta_{7, N}=0 \quad \text { and } \quad \zeta_{8, N}=0 .
\end{aligned}
$$

To obtain the optimality conditions, we take the variation with respect to control $\left(u_{i}^{*}, v_{i}^{*}\right)$ and set it equal to zero

$$
\begin{aligned}
& \frac{\partial H}{\partial u_{i}}=\tau_{1} u_{i}-\zeta_{1, i+1} V_{i}^{M}-\zeta_{2, i+1} V_{i}^{W}+\zeta_{3, i+1} V_{i}^{M}+\zeta_{4, i+1} V_{i}^{W}=0 \\
& \frac{\partial H}{\partial v_{i}}=\tau_{2} u_{i}+\zeta_{3, i+1} D_{i}^{M}+\zeta_{4, i+1} D_{i}^{W}-\zeta_{5, i+1} D_{i}^{M}-\zeta_{6, i+1} D_{i}^{W}=0 .
\end{aligned}
$$

Then we obtain the optimal control

$$
\begin{aligned}
& u_{i}^{*}=\frac{V_{i}^{M}\left(\zeta_{1, i+1}-\zeta_{3, i+1}\right)+D_{i}^{W}\left(\zeta_{2, i+1}-\zeta_{4, i+1}\right)}{\tau_{1}}, \\
& v_{i}^{*}=\frac{D_{i}^{M}\left(\zeta_{5, i+1}-\zeta_{3, i+1}\right)+D_{i}^{W}\left(\zeta_{6, i+1}-\zeta_{4, i+1}\right)}{\tau_{2}} .
\end{aligned}
$$


By the bounds in $\mathcal{U}_{\mathrm{ad}}$, it is easy to obtain $\left(u_{i}{ }^{*}, v_{i}{ }^{*}\right)$ in the following form:

$$
\begin{aligned}
& u_{i}^{*}=\min \left\{\max \left\{\frac{V_{i}^{M}\left(\zeta_{1, i+1}-\zeta_{3, i+1}\right)+D_{i}^{W}\left(\zeta_{2, i+1}-\zeta_{4, i+1}\right)}{\tau_{1}}, u_{\min }\right\}, u_{\max }\right\}, \\
& v_{i}^{*}=\min \left\{\max \left\{\frac{D_{i}^{M}\left(\zeta_{5, i+1}-\zeta_{3, i+1}\right)+D_{i}^{W}\left(\zeta_{6, i+1}-\zeta_{4, i+1}\right)}{\tau_{2}}, u_{\min }\right\}, u_{\max }\right\},
\end{aligned}
$$

for $i=0, \ldots, N-1$.

\section{Numerical simulation}

We present now the obtained numerical simulations in the case when the studied domain represents cities or towns. We use data from Table 2. The HCP gives different statistics according to the age groups of the population. For example, the percentage of divorce is $30 \%$ for couples with less than 5 years of marriage and 3\% for married couples who have exceeded 20 years of marriage. There are $76.2 \%$ of single people for the age group 20 to 24 and $54 \%$ for the group of 25 to 29 year-olds. Generally, the HCP gives the average sum of marriage rates between different population categories $\alpha_{1}, \alpha_{2}, \ldots, \beta_{5}$. One of the main objectives of our work is to estimate the values of the parameters $\alpha_{1}, \ldots, \lambda_{3}$ as precisely as possible, for example, by using the least squares method and adjusting results coming from a statistical study that is planned to be done in subsequent work. The multi-points boundary value problems are solved using an iterative method where at instant $i$, the states $V_{i}^{M}, V_{i}^{W}, M_{i}^{M}, M_{i}^{W}, D_{i}^{M}, D_{i}^{W}, W_{i}^{M}$ and $W_{i}^{W}$ with an initial guess are obtained based on a progressive schema in time, and their adjoint variables $\zeta_{k, i}, k=1,2, \ldots, 8$, are obtained based on a regressive schema in time because of the transversality conditions. Then we update the optimal controls values (18), (19) using the values of state and costate variables obtained in the previous steps. Finally, the previous steps are executed until we reach a tolerance criterion.

Considering the critical level of control, we give an optimal control sufficient to reduce the number of virgin and divorced individuals and to increase the number of married individuals.

In the following, we can see that the optimal control function has a very desirable effect upon the population of virgin and divorced which decreases, while the married population increases for almost the entire length of process.

The graphs below allow us to compare changes in the number of virgin, divorced and married individuals before and after the introduction of control. The time evolution of the respective populations with and without control is displayed in Figures 2 and 3, respectively.

In a population of 1,530 individuals, the number of uncontrolled virgin men decreases to 200 virgin men. On the other hand, by applying the control law, this number decreases

Table 2 The description of parameters used for the definition of discrete time systems (9)-(16)

\begin{tabular}{lllllllll}
\hline$V_{0}^{M}$ & $V_{0}^{W}$ & $M_{0}^{M}$ & $M_{0}^{W}$ & $D_{0}^{M}$ & $D_{0}^{W}$ & $W_{0}^{M}$ & $W_{0}^{W}$ & $\alpha_{1}$ \\
450 & 460 & 250 & 200 & 40 & 80 & 20 & $\beta_{4}$ & $\beta_{5}$ \\
$\alpha_{2}$ & $\alpha_{3}$ & $\alpha_{4}$ & $\beta_{1}$ & $\beta_{2}$ & $\beta_{3}$ & $\lambda_{1}$ \\
0.003 & 0.002 & 0.008 & 0.03 & 0.04 & 0.02 & 0.008 & 0.01 & 0.005 \\
$\lambda_{2}$ & $\lambda_{3}$ & $A_{1}$ & $A_{2}$ & $A_{3}$ & $\tau_{1}$ & $\tau_{2}$ & \\
0.001 & 0.003 & 0.5 & 0.1 & 0.1 & $1.6 .10^{14}$ & $1.2 .10^{13}$ & \\
\hline
\end{tabular}



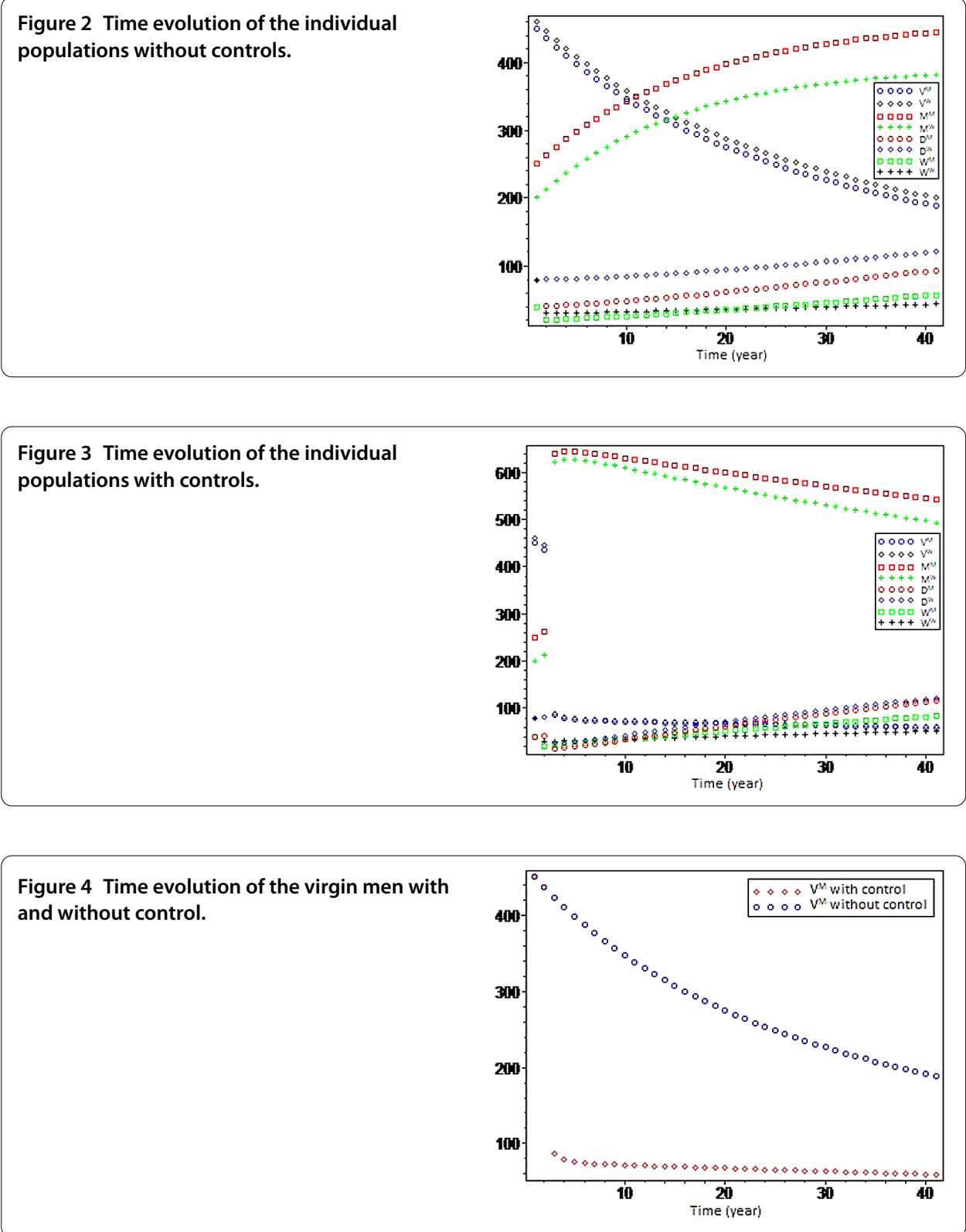

rapidly to reach out the end of the companion awareness of 20 virgin men. This result is similar to virgin women as shown in Figures 4 and 5.

Figures 6 and 7 show that the number of married men and women grows after 5 years and reaches a maximum value of about 650 for married men and 630 for married women, then it declines to 550 for men and 500 for women. This decline is due to the fact that the number of divorced men and women develops after five years, but it is still quite high as far as the uncontrolled number of married men and women, with a maximum value of 400 for men and 380 for women.

Figures 8 and 9 also show the effect of applying the control law by indicating that the number of divorced individuals decreases faster at the beginning of treatment. Then we notice that the number increases and we can justify it by the fact that the number of divorced individuals is proportional to the number of married individuals. Since the number 


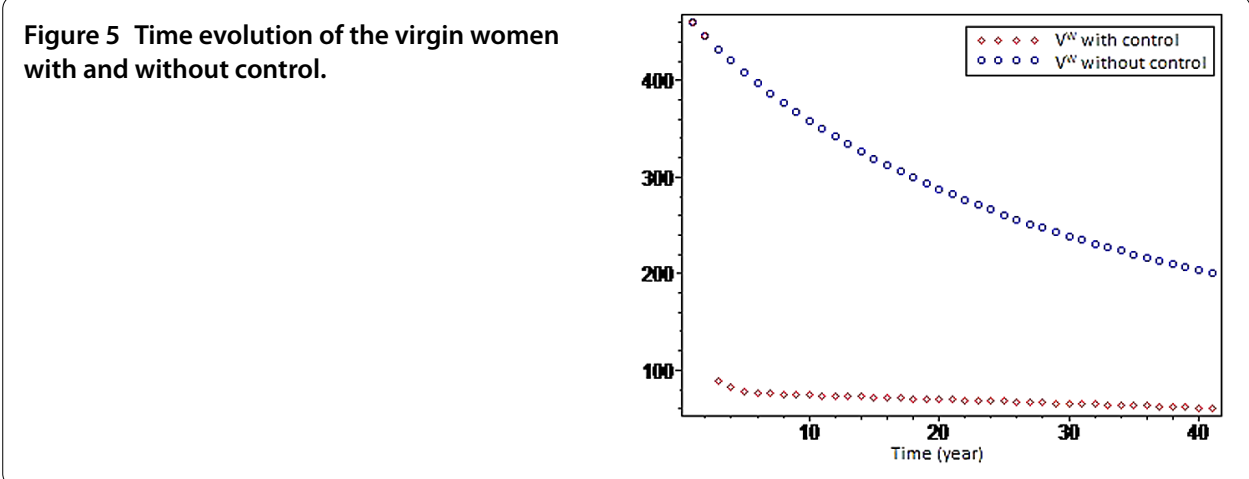

Figure 6 Time evolution of the married men with and without control.
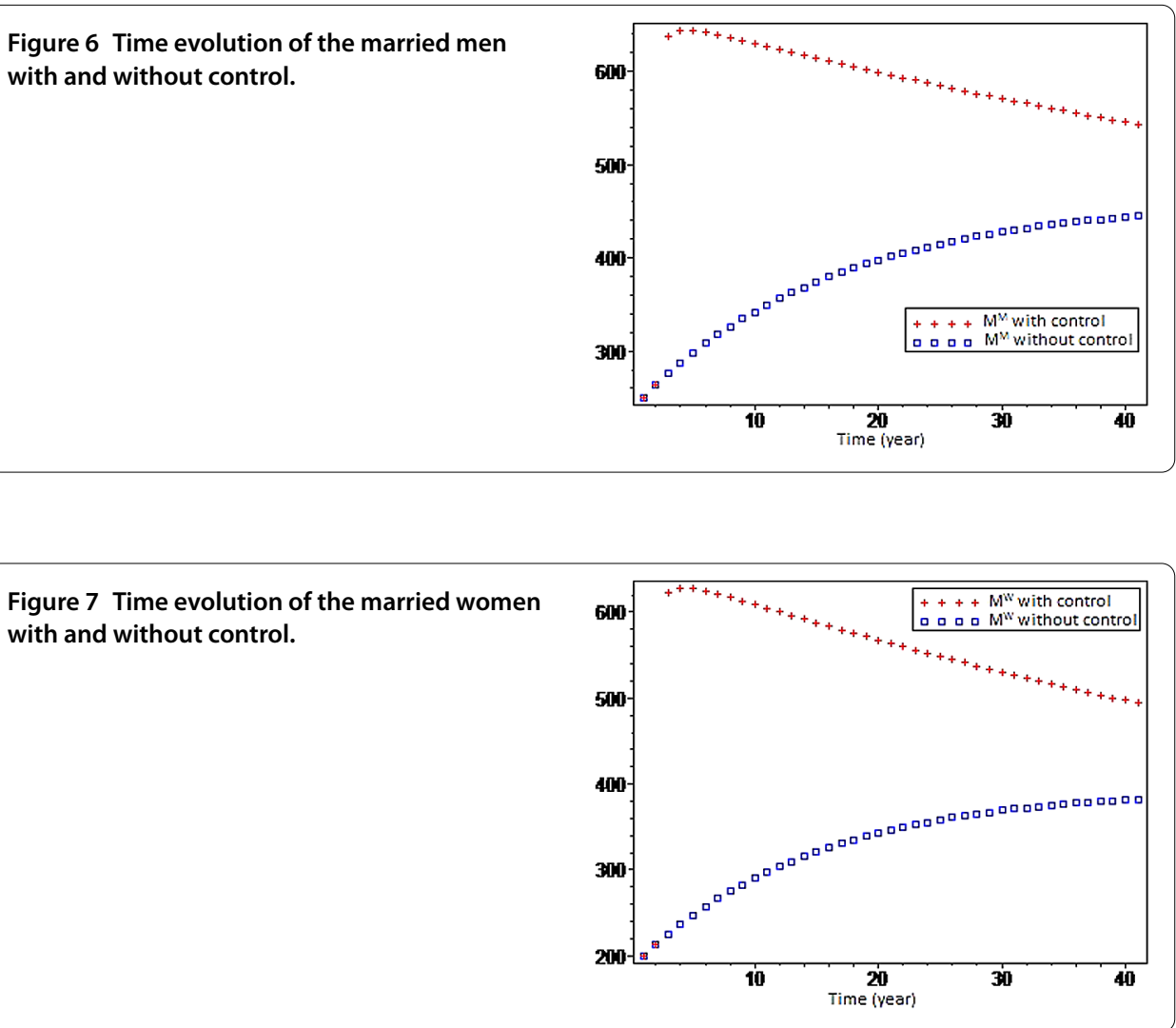

Figure 8 Time evolution of the divorced men with and without control.

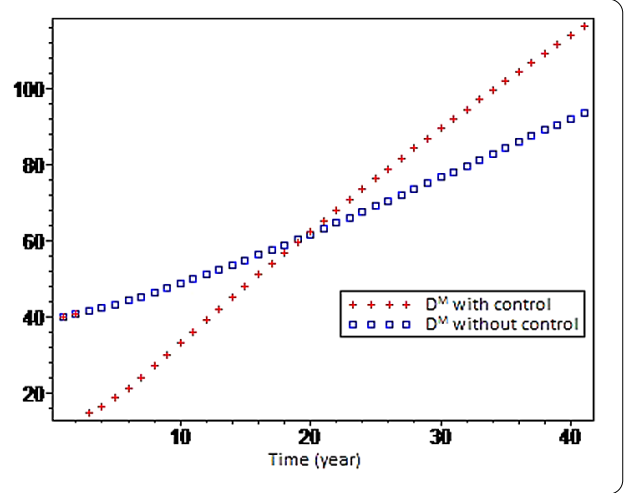




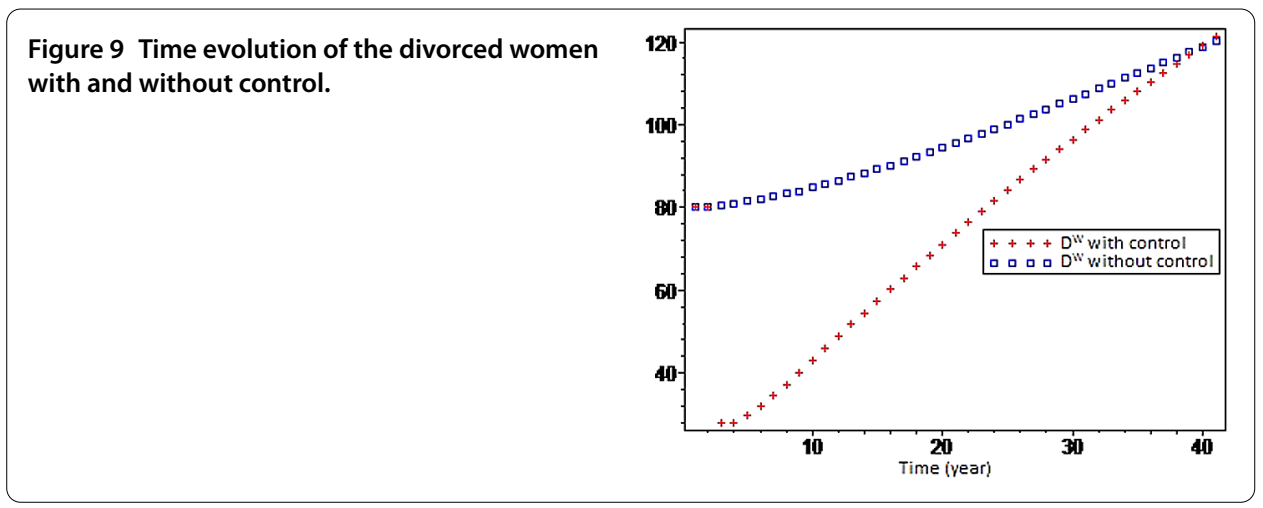

Figure 10 Time evolution of control $u^{*}$.

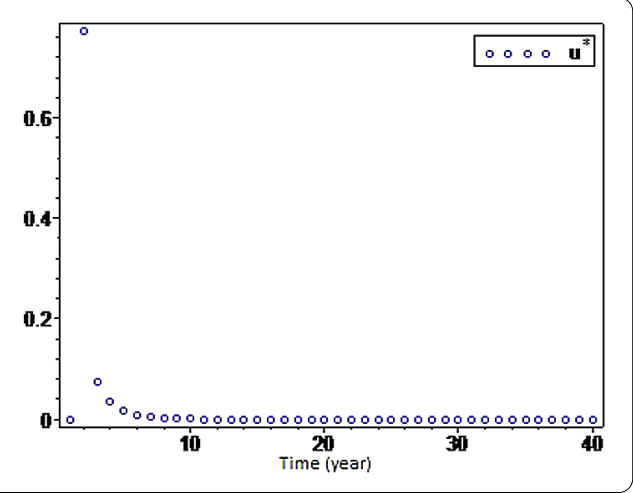

Figure 11 Time evolution of control $v^{*}$.

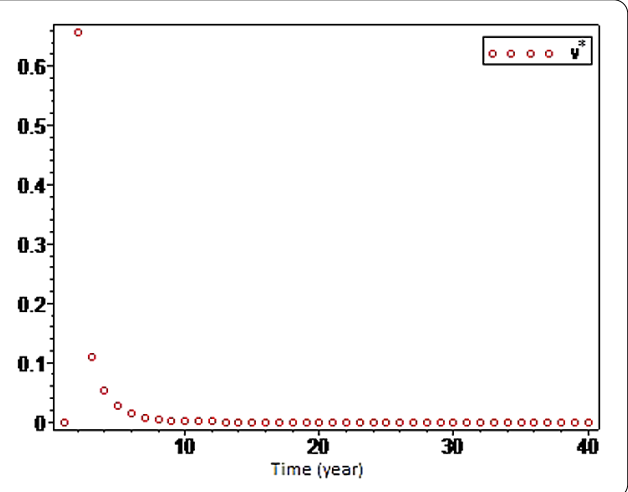

of married individuals increases along the treatment, it is normal that the number of divorced individuals also increases.

Finally, Figures 10 and 11 display the time evolution of the optimal control $\left(u^{*}, v^{*}\right)$.

\section{Conclusions}

In this paper, we consider a discrete-time marital status model, we treat the modeling and control of a system that describes the case of monogamous marriage. An optimal control is investigated to reduce the number of virgin, divorced population and to increase the married one. The first control introduced the benefits of an awareness campaign to educate virgin men and women about the benefits of marriage for individuals and for the society, and the second control characterized the legal procedures, administrative complications and the heavy financial and social consequences of divorces. A discrete version 
of Pontryagin's maximum principle is used to analyze the optimal control problem, and a numerical simulation is given to illustrate the obtained results.

\author{
Competing interests \\ The authors declare that they have no competing interests.
}

\title{
Authors' contributions
}

All authors contributed equally to the writing of this paper. All authors read and approved the final manuscript.

\section{Author details}

${ }^{1}$ Laboratory of Modelling, Analysis, Control and Statistics, Department of Mathematics and Computer Science, Faculty of Sciences Ain Chock, Hassan II University of Casablanca, B.P 5366 Maarif, Casablanca, Morocco. ${ }^{2}$ Laboratory of Analysis Modelling and Simulation, Department of Mathematics and Computer Science, Faculty of Sciences Ben M'sik, Hassan II University of Casablanca, B.P 7955 Sidi Othman, Casablanca, Morocco. ${ }^{3}$ Department of Mathematics, Faculty of Sciences, University Ibn Tofail, B.P 133, Kénitra, Morocco.

\section{Publisher's Note}

Springer Nature remains neutral with regard to jurisdictional claims in published maps and institutional affiliations.

Received: 7 July 2017 Accepted: 6 October 2017 Published online: 23 October 2017

\section{References}

1. Canale, RP: An analysis of models describing predator-prey interaction. Biotechnol. Bioeng. XII, 353-358 (1970)

2. Conway, ED, Smoller, JA: Global analysis of a system of predator-prey equations. SIAM J. Appl. Math. 46(4), 630-642 (1986)

3. Galbraith, JK: The predator state. Mother Jones, May/June (2006)

4. Yan, J, Li, C, Chen, X, Ren, L: Dynamic complexities in 2-dimensional discrete-time predator-prey systems with Allee effect in the prey. Discrete Dyn. Nat. Soc. 2016, 4275372 (2016)

5. Agusto, FB: Optimal isolation control strategies and cost effectiveness analysis of a two-strain avian influenza model. Biosystems 113(3), 155-164 (2013)

6. Allen, LJ: Some discrete-time SI, SIR, and SIS epidemic models. Math. Biosci. 124(1), 83-105 (1994)

7. Birkhoff, G, Rota, GC: Ordinary Differential Equations, 4th edn. Wiley, New York (1989)

8. Brauer, F, Castillo-Chavez, C: Mathematical Models in Population Biology and Epidemiology. Texts in Applied Mathematics, vol. 40. Springer, New York (2001)

9. Cavalli Sforza, LL, Feldman, MW: The application of molecular genetic approaches to the study of human evolution. Nat. Genet. 33, 266-275 (2003)

10. Meyer, UA, Zanger, UM: Molecular mechanisms of genetic polymorphisms of drug metabolism. Annu. Rev. Pharmacol. Toxicol. 37, 269-296 (1997)

11. Nicholson, JK: Global systems biology, personalized medicine and molecular epidemiology. Mol. Syst. Biol. 2, 52 (2006)

12. Bennetta, K, Morgan, $\mathrm{K}$ : Health, social functioning and marital status: stability and change among elderly recently widowed women. Int. J. Geriatr. Psychiatry 7(8), 13-817 (1992)

13. Goodwin, PY, Mosher, WD, Chandra, A: Marriage and cohabitation in the United States: a statistical portrait based on cycle 6 (2002) of the National Survey of Family Growth. In: Vital Health Stat. 23, vol. 28. National Center for Health Statistics (2010)

14. Jedlicka, D: Affinographs a Dynamic Method for Assessment of Individuals, Couples, Families, and Households. Springer, Berlin (2011)

15. Karney, BR, Bradbury, TN: Contextual influences on marriage: implications for policy and intervention. Curr. Dir. Psychol. Sci. 14, 171-174 (2005)

16. Keyfitz, N, Flieger, W: Populations: Fact and Methods of Demography. Freeman, San Francisco (1971)

17. Umberson, D: Gender, marital status and the social control of health behavior. Soc. Sci. Med. 34(8), 907-917 (1992)

18. Usdansky, ML, London, AS, Wilmoth, JM: Veteran status, race, ethnicity, and marriage among fragile families. J. Marriage Fam. 71, 768-786 (2009)

19. Waldron, I, Weiss, CC, Hughes, ME: Marital status effects on health: are there differences between never married women and divorced and separated women? Soc. Sci. Med. 45(9), 1387-1397 (1997)

20. Zax, JS, Flueck, DW: Marriage, divorce, income and marriage incentives. Working Paper, Department of Economics, University of Colorado, Boulder, Boulder (2003)

21. High Commission for Planning of Morocco: Marriage and divorce Moroccan woman evolutionary trends. National Documentation Center (2014). http://www.hcp.ma/Mariage-et-divorce-de-la-femme-marocaine-Tendances-devolution_a1261.html

22. El-Alami Laaroussi, A, Rachik, M, Elhia, M: An optimal control problem for a spatiotemporal SIR model. Int. J. Dyn. Control (2016). doi:10.1007/s40435-016-0283-5

23. Dabbs, K: Optimal control in discrete pest control models. Honors Thesis Projects, University of Tennessee (2010)

24. Hattaf, K, Rachik, M, Saadi, S, Yousfi, N: Optimal control of infection model. Appl. Math. Sci. 3(20), 949-958 (2009)

25. Joshi, HR: Optimal control of an HIV immunology model. Optim. Control Appl. Methods 23(4), 199-213 (2002)

26. Laarabi, H, Rachik, M, El Kahlaoui, O, Labriji, E: Optimal vaccination strategies of an SIR epidemic model with a saturated treatment. Univers. J. Appl. Math. 1(3), 185-191 (2013)

27. Lhous, M, Rachik, M, Larrache, A: Free optimal time control problem for a SEIR-epidemic model with immigration of infective. Int. J. Comput. Appl. 159(3), 1-5 (2017) 
28. Wandi, D, Hendon, R, Cathey, B, Lancaster, E, Germick, R: Discrete time optimal control applied to pest control problems. Involve 7(4), 479-489 (2014)

29. Pontryagin, LS, Boltyanskii, VG, Gamkrelidze, RV, Mishchenko, EF: The Mathematical Theory of Optimal Processes. Wiley, New York (1962)

Submit your manuscript to a SpringerOpen ${ }^{\circ}$ journal and benefit from:

- Convenient online submission

$\checkmark$ Rigorous peer review

- Open access: articles freely available online

- High visibility within the field

- Retaining the copyright to your article

Submit your next manuscript at $\boldsymbol{\triangleright}$ springeropen.com 